\title{
Robust Flatness-based Multi-controllers Approach
}

\author{
Hajer GHARSAllaOUI ${ }^{1,2}$, Mounir AYADI ${ }^{1}$, Mohamed BENREJEB ${ }^{1}$, Pierre BORNE $^{2}$ \\ ${ }^{1}$ Unité de Recherche LA.R.A Automatique, Ecole Nationale d'Ingénieurs de Tunis \\ B.P. 37, le Belvédère, 1002 Tunis, Tunisie, \\ hajer.gharsallaoui@voila.fr,mounir.ayadi@enit.rnu.tn,mohamed.benrejeb@enit.rnu.tn \\ ${ }^{2}$ LAGIS, École Centrale de Lille \\ B.P. 48, Villeneuve d'Ascq, 59651 Lille, France, \\ pierre.borne@ec-lille.fr
}

\begin{abstract}
In this paper, a robust fault tolerant control reconfiguration is proposed. For identified models whose parameters change according to operating conditions, it is convenient to design a reconfiguration control strategy. This approach switches on-line the right controller to achieve the same performances for a given objective. This reconfiguration strategy is based on robust flatness-based switching control and developed in discrete-time framework in order to track a reference trajectory starting from a flat output variable. For each model, a corresponding flatness-based controller is designed and consequently, a multi-controller structure is obtained. The switching process is based on the minimization of a criterion. The performances obtained by switching in terms of tracking trajectory and disturbance fault rejection are discussed in this paper. The stability of the corresponding switched discrete-time linear systems is given.
\end{abstract}

Keywords: Flatness, tracking trajectory, robustness, switching, multi-controllers, reconfiguration.

\section{Introduction}

Associated with the increasing demands for higher system performance and product quality on the one side and more cost efficiency in the other side, the complexity and the automation degree of technical processes are continuously growing. However, conventional feedback control design for a complex system may result in an unsatisfactory performance, or even in instability, in the event of malfunctions in actuators, sensors or other system components. In order to overcome these limitations, modern complex systems use sophisticated controllers which are developed with fault accommodation and tolerance capabilities, in order to meet reliability and performance requirements.

Originated in the early 70 's, the model-based reconfiguration control technique is a kind of active approaches to achieve fault tolerant control for dynamic systems which has been developed remarkably since then. Its efficiency in detecting faults in a system has been demonstrated by a great number of successful applications in industrial processes and automatic control systems.

Although developed for different purposes by means of different techniques, all model-based fault tolerant control system are common in the explicit use of a process model, based on which algorithms are implemented for processing data that are on-line collected and recorded during the system operation.

In this way, general multiple model-based control approaches have been developed in last decade, [3]-[5]-[8]-[10]-[21]. Thus, in the literature, there are many strategies that take in consideration the multi-models methods used for reconfiguration of control law and diagnosis purposes, [7]-[14]-[18]-[20]-[22]. Furthermore, Multiple Models (MM) method is proposed in [16]. In addition, controller switching approach represents a class of active fault tolerant control detailed in [17]. Moreover, Multiple Models Switching and Tuning (MMST) approach that concerns more particularly the reconfigurable control method is proposed in [4]-[9]-[19]-[20]-[24][25]-[26].

In this paper, for a Single Input Single Output (SISO) nonlinear process with multiple operating modes, a robust control reconfiguration strategy, based on switching control, is used to ensure stability and desired performances. The multi-model flatnessbased control is based on switching between identified operating modes. The minimization of criterion value, based on a difference between the estimated output of each model and the real system output, is generated. The LMI tools which are based on quadratic Lyapunov criteria in order to guaranty the stability of the global system are used. The performances obtained by switching in terms 
of tracking trajectory and disturbance rejection are discussed in this paper and the stability of the corresponding switched discrete-time linear systems is given. The case of thermal process is studied.

\section{Reconfiguration Fault Tolerant Control Approach}

\subsection{Description}

Each controller of the proposed approach is designed using a robust pole assignment proposed in [1]. This control law presents a good performance in term of robustness and guarantees closed-loop performance in case of small parameters uncertainties. Operating mode detection and active fault tolerant control structures are introduced in this paper to deal with an active supervisory control integrating an additional detection reconfiguration loop. In the presence of exogenous fault, the active method of robust control reconfiguration is based on using an indirect switching strategy dealing with the selection of the right controller.

The controller selection strategy is based on the minimization of the error between the output of each model and the real output of the system. Each controller $C_{j}$ is designed especially for a given process operating mode $G_{j}$, [7]. In order to determine when and into which controller we should switch, a detection method is detailed below:

- the model $G_{j}$ is controlled by the signal $u_{r}$, which is issued from the active controller, controller corresponds to the argument of the smallest criteria $J_{j}$ value which expression is given in next paragraph,

- the detection rule gives the decision of the correspond desired trajectory $z_{k, j}^{d}$ calculated for active operator mode.

\subsection{Residual evaluation and detection rule}

For $j$ models, the state space representation in a discrete-time framework is given by:

$\left\{\begin{array}{l}x_{k+1, j}=\mathbf{A}_{j} x_{k, j}+\mathbf{B}_{j} u_{k, j} \\ y_{k, j}=\mathbf{C}_{j} x_{k, j}\end{array}\right.$

where $\mathrm{A}_{j}, \mathrm{~B}_{j}$, and $\mathrm{C}_{j}$, , represent the system matrices of state space.

Among the existing model-based fault tolerant control reconfiguration schemes, we find the so-called observer-based technique. This technique has been developed in the framework of the well established advanced control theory, where powerful tools for designing observers for efficient and reliable algorithms for data processing aiming at reconstructing process variable are available.

In order to receive this information, a comparison of measured process variables (output signals) with their estimates delivered by the process models will then be made. The difference between the measured process variables and their estimates is called residue. Roughly speaking a residual signal carries the most important message for a successful fault reconfiguration control; if residual is different to zero then fault otherwise fault free.

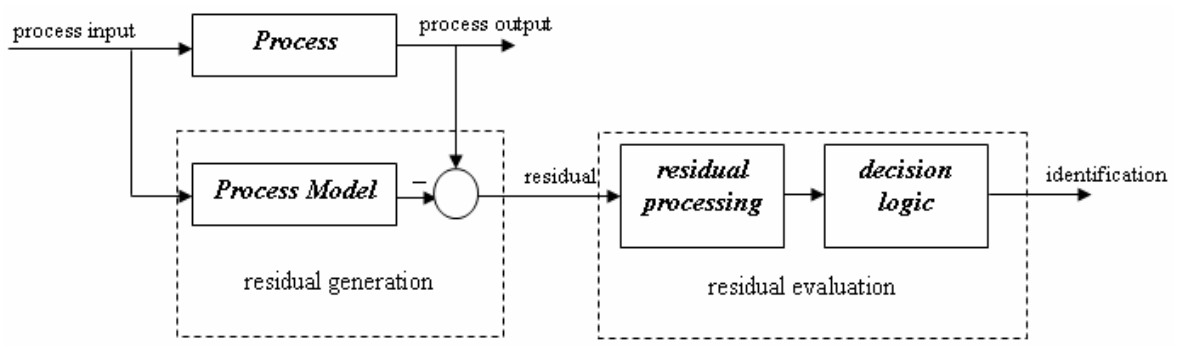

Figure 1. Schematic description of the model-based reconfiguration

- a residual evaluation is calculated for each model output,

- a mode isolation based on the detection rule decides if the process $G_{j}$ is in operating mode with sampling period $T_{e}$. A minimization of a criteria $J_{j}$ is carried out and consequently the selected
The procedure of creating the estimates of the process outputs and building the difference between the process outputs and their estimates is called residual generation. Correspondingly, the process model and the comparison unit build the so-called residual generator, as shown in Figure 1. 
The associated Lunberger observers are then given as follows by:

$\left\{\begin{array}{l}\hat{x}_{k+1, j}=\mathbf{A}_{j} \hat{x}_{k, j}+\mathbf{B}_{j} u_{k, j}+\mathbf{L}_{j}\left(y_{k}-\hat{y}_{k, j}\right) \\ \hat{y}_{k, j}=\mathbf{C}_{j} \hat{x}_{k, j}\end{array}\right.$

The observers' banc is illustrated in Figure 2.

The residual value is expressed by:

$\varepsilon_{j}=y_{k}-\hat{y}_{k, j}$

which will be used to define the detection criterion.

$\mathrm{L}_{j}$ are the observer gains for which the eigenvalues of $\left(\mathrm{A}_{j}-\mathrm{L}_{j} \mathrm{C}_{j}\right)$ are stable, [6].

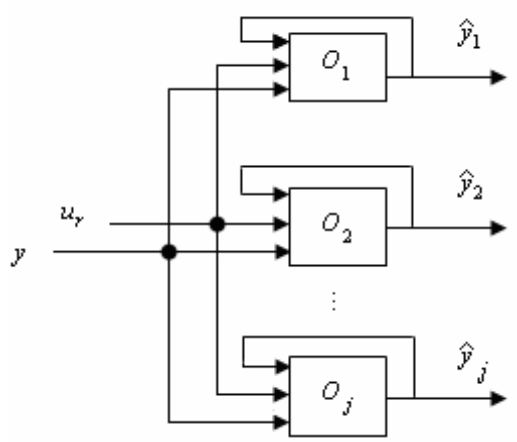

Figure 2. Observers' banc schematic

The detection rule is calculated online based on the minimization of the residue procession criterion value and that generates the minimum argument in order to choose the adequate active controller for the current operating mode which behavior represents the best the real plant model.

In this paper, an Operating Mode Detection and Accommodation (OMDA) structure [7] is introduced to deal with an active supervisory control including flatness-based multicontrollers, (see Figure 3).

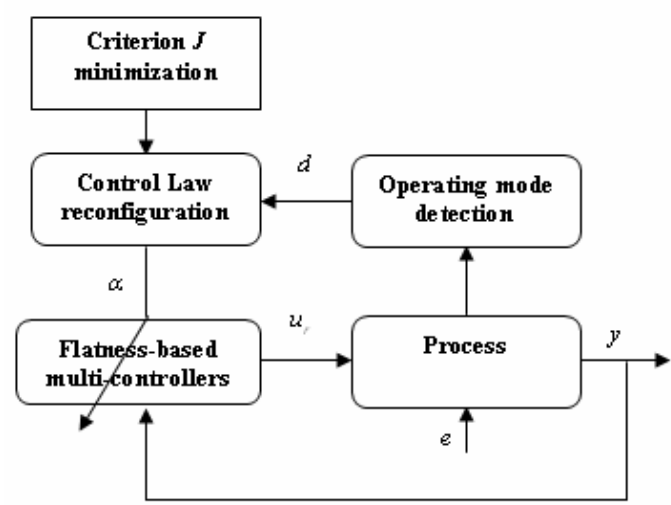

Figure 3. Structure of Operating Mode Detection and Reconfiguration scheme
In the presence of exogenous events $e$, the active method of robust control reconfiguration is based on the use of an indirect switching strategy dealing with the detection of the switching time and the selection of the right controller.

Then, a multi-controller structure is required to control a process with several Operating Modes.

The monitoring structure consists of two combined blocks, one for the model-based detection, which allows detecting a given process operating mode $(d)$, and the other one for the accommodation decision $(\alpha)$, which selects the right controller. The controller selection strategy is based on the minimization of a criterion, which will be described bellow. Remember that each controller $C_{j}$ is designed specifically for a given process operating mode $G_{j}$. An operating mode matrix is defined such as:

$M=\left\{M_{i, j}=\left(C_{i}, G_{j}\right),(i, j) \in I^{2}\right\}$

where $I$ is a finite set of integers $I=\{1,2, \ldots, g\}$. If $j=i$, then $M_{i, j}$ represents the $i^{\text {th }}$ fitted operating mode, i.e., the controller matches the OM exactly, otherwise if $j \neq i$, then $M_{i, j}$ corresponds to one of the non fitted operating modes, i.e., the controller does not match the OM.

In order to determine when and to which controller one should switch, a detection method is detailed in the following.

The detector consists of three functions:

1. simulation of the models $G=\left\{G_{j}, j \in\{1, \ldots, g\}\right\}$ controlled by the signal $u_{r}$, which is issued from the active controller;

2. evaluation for each model error output according to the fixed criterion mentioned in equation (5);

3. mode isolation based on the detection rule.

For each operating mode, $j \in I$ a criterion is associated and expressed by:

$J_{j}=\frac{1}{N-1} \sum_{n=0}^{N-1} \varepsilon_{j, n}^{2}(k)$

where $N$ is the size of the sliding window, $\varepsilon_{j, n}(k)$ is the $j^{\text {th }}$ modelling error given by:

$\varepsilon_{j, n}(k)=y(k-n)-y_{j}(k-n)$ 
The Multi-model Output Recursive Square Error (MORSE) criterion [18]:

$$
J(k)=\left[\begin{array}{lllll}
J_{1}(k) & J_{2}(k) & \cdots & J_{j}(k) & \cdots
\end{array}\right]
$$

is computed with the recursive formula obtained from the relation (5):

$$
J_{j}(k)=J_{j}(k-1)+\frac{1}{N-1}\left(\varepsilon_{j, n}^{2}(k)-\varepsilon_{j, n}^{2}(k-N)\right)
$$

while $k<N$, the cumulative summation of the square errors of the relation (8), is used to initialize the criterion $J_{j}$.

The couple $\left(d, t_{d}\right)$ defines the detection test, which describes each operation mode $d(k)$ detected and the detection time $t_{d}(k)$.

The detection rule is computed on-line by:

$$
d(k)=\left\{P=G_{m}, m=\underset{1 \leq j \leq g}{\arg \min } J_{j}(k)\right\}
$$

This rule decides that the process $P$ is operating in the $m^{\text {th }}$ mode $G_{m}$ sampled at the control sampling period $T_{e}$. At each sampling period, a minimization of the criterion $J_{j}(k)$ is carried out. The selected controller corresponds to the argument of the smallest $J_{j}$.

In this paper, only one objective is considered, i.e., regulating the output of a SISO system to a reference input signal. The accommodation vector $\alpha$ is a piecewise continuous switching signal which represents the series of the successive activated controllers. This is the set of decisions which selects the $j^{\text {th }}$ controller corresponding to the detected mode and whose the criterion $J_{j}$ is the smallest.

\subsection{Stability Analysis}

The stability analysis of a switching control strategy consists on two steps, [7]:

stability analysis of each subsystem, i.e., each controller must asymptotically stabilize each process operating mode,

stability analysis of the overall system for arbitrary switching signals.

To guarantee the stability of the systems, Linear Matrix Inequalities must be solved. A common Lyapunov function must be found for the switched linear system, [11].
In the case of Linear Time Invariant (LTI) systems, the quadratic stability is determined by Lyapunov function as: $V(x, k)=x_{k}^{T} \mathbf{P} x_{k}>0$ and $\quad \mathbf{P}>0$ to study the stability of systems of the form are:

$x_{k+1}=\mathbf{A} x_{k}$

to guarantee the asymptotic stability of systems in accordance with Lyapunov theory, the function $V(x, k)$ must be such that:

$V(x+1, k+1)-V(x, k)<0$

then the system (10) is quadratic stable if the inequality:

$\mathbf{A}^{T} \mathbf{P A}-\mathbf{P}<0$

is verified.

The equivalence is to express the quadratic stability using the Linear Matrix Inequalities represented by the following relations:

If $\mathbf{P}=\mathbf{P}^{T}>0$, so:

$$
\mathbf{P}-\mathbf{A}^{T} \mathbf{P A}>0 \Leftrightarrow\left(\begin{array}{cc}
\mathbf{P} & \mathbf{A}^{T} \mathbf{P} \\
\mathbf{P A} & \mathbf{P}
\end{array}\right)>0
$$

Using these linear matrix inequalities, we can guaranty the stability of the switched system. Moreover, using the LMI tools we calculate the observer's gains.

\section{Design of Flatness-based Controller}

In this part, a flatness-based RST controller design is presented to be used in the switching multi-controllers approach in order to track a non constant reference trajectory.

\subsection{Discrete-time flat output}

The discrete-time SISO system, described by the following equation:

$A(q) y_{k}=B(q) u_{k}$

where $y_{k}$ and $u_{k}$ represent respectively the output and the control signals, is flat if it is controllable. The discrete-time flat output $z_{k}$ is then given by [1]:

$z_{k}=N(q) y_{k}+D(q) u_{k}$

where $N(q)$ and $D(q)$ are polynomials which represent the solutions of the Bezout equation: 


$$
A(q) D(q)+B(q) N(q)=1
$$

The flat output $z_{k}$ on which depends the output $y_{k}$ and the control $u_{k}$, can be also seen as being the partial state of a linear system, [1]-[2]-[23]:

$u_{k}=A(q) z_{k}$

$y_{k}=B(q) z_{k}$

The knowledge of the flat output $z_{k}$ and its future values makes it possible the determination of the open-loop control $u_{k}$ of the system.

\subsection{Trajectories planning}

The objective of the trajectories planning is to determine an open-loop control $u^{d}(t)$, bringing a given system, from a certain initial state in a known final state [12]:

$$
\begin{aligned}
u^{d}(t) & =\mathbf{B}\left(z^{d}(t), \ldots, z^{d(\alpha+1)}(t)\right), \\
y^{d}(t) & =\mathbf{C}\left(z^{d}(t), \ldots, z^{d(\sigma)}(t)\right)
\end{aligned}
$$

$z^{d}$ is the desired trajectory for the continuous-time flat output that must be $\sup (\alpha+1, \sigma)$ time continuously derivable.

If the objective is to reach two equilibrium points of balance $\left(u\left(t_{0}\right), y\left(t_{0}\right), z\left(t_{0}\right)\right)$ and $\left(u\left(t_{f}\right), y\left(t_{f}\right), z\left(t_{f}\right)\right), t_{0}$ and $t_{f}$ are the two instants known in advance, it is necessary to solve the problem of generation of trajectory off line.

Different steps of desired trajectory $y_{k}^{d}$ generation in discrete-time formalism are described in [1]-[2], where:

$$
y_{k}^{d}=B(q) z_{k}^{d}
$$

where $z_{k}^{d}$ are the values of the continuous-time trajectory $z^{d}(t)$ at the $k$ sampling instant.

\subsection{Flatness-based RST controller design}

The realization of a flatness-based RST controller is carried out by considering the method of direct calculation of the state vector $Z_{k}=\left(\begin{array}{llll}z_{k} & z_{k+1} & \cdots & z_{k+n-1}\end{array}\right)^{T}$, suggested in [1]-[2]-[23].
From the relations (17) and (18), we can determine the representation of system state in its controllable form:

$\left\{\begin{array}{l}Z_{k+1}=\mathbf{A} Z_{k}+\mathbf{B} u_{k}, \\ y_{k}=\mathbf{C} Z_{k},\end{array}\right.$

with:

$\mathbf{A}=\left(\begin{array}{ccccc}0 & 1 & 0 & \cdots & 0 \\ 0 & \ddots & 1 & \ddots & \vdots \\ \vdots & \ddots & \ddots & \ddots & 0 \\ 0 & \cdots & 0 & 0 & 1 \\ -a_{0} & -a_{1} & \cdots & -a_{n-2} & -a_{n-1}\end{array}\right), \quad \mathbf{B}=\left(\begin{array}{c}0 \\ 0 \\ \vdots \\ 0 \\ 1\end{array}\right)$
$\mathbf{C}=\left(\begin{array}{llll}b_{0} & b_{1} & \cdots & b_{n-1}\end{array}\right)$

From this representation, we can write:

$Z_{k}=O_{(\mathbf{A}, \mathbf{C})}^{-1}\left(Y_{k}-M_{(\mathbf{A}, \mathbf{B}, \mathbf{C})} U_{k}\right)$

where the observability matrix is:

$O_{(\mathbf{A}, \mathbf{C})}=\left(\begin{array}{c}\mathbf{C} \\ \mathbf{C A} \\ \vdots \\ \mathbf{C A}^{n-1}\end{array}\right)$

and the controllability matrix $M_{(A, B, C)}$ given by:

$$
M_{(\mathbf{A}, \mathbf{B}, \mathbf{C})}=\left(\begin{array}{cccc}
0 & \cdots & \cdots & 0 \\
\mathbf{C B} & \ddots & & \vdots \\
\mathbf{C A B} & \mathbf{C B} & \ddots & \vdots \\
\vdots & \ddots & \ddots & 0 \\
\mathbf{C A}^{n-2} \mathbf{B} & \cdots & \mathbf{C A B} & \mathbf{C B}
\end{array}\right)
$$

The flatness-based control law is given by the following relation:

$u_{k}=K(q) z_{k}^{d}+(a-k) Z_{k}$

where $a$ and $k$ are two constant vectors constituted by the coefficients of the $A(q)$ and $K(q)$ polynomials:

$a=\left(\begin{array}{llll}a_{0} & a_{1} & \ldots & a_{n-1}\end{array}\right), k=\left(\begin{array}{llll}k_{0} & k_{1} & \ldots & k_{n-1}\end{array}\right)$

and $K(q)$ is a polynomial containing the closed loop poles. The realisable structure of RST flatness-based controller can be then obtained by:

$S\left(q^{-1}\right) u_{k}=K(q) z_{k}^{d}+R\left(q^{-1}\right) y_{k}$

where:

$$
R\left(q^{-1}\right)=-(a-k) \mathbf{A}^{n-1} O_{(\mathbf{A}, \mathrm{C})}^{-1} Q
$$




$$
\begin{aligned}
S\left(q^{-1}\right) & =1+(a-k) \cdot \\
& \cdot\left(\mathbf{A}^{n-1} O_{(\mathbf{A}, \mathbf{C})}^{-1} M_{(\mathbf{A}, \mathbf{B}, \mathbf{C})}-\right. \\
& \left.-\left(\begin{array}{llll}
\mathbf{A}^{n-2} \mathbf{B} & \ldots & \mathbf{B}
\end{array}\right)\right) Q \\
Q^{*} & =\left(\begin{array}{lllll}
q^{-(n-1)} & q^{-(n-2)} & \ldots & q^{-1}
\end{array}\right)^{T} \\
Q & =\left(\begin{array}{lllll}
q^{-(n-1)} & q^{-(n-2)} & \ldots & q^{-1} & 1
\end{array}\right)^{T}
\end{aligned}
$$

The closed-loop dynamics is defined by the tracking polynomial $K(q)$ such as:

$$
A\left(q^{-1}\right) S\left(q^{-1}\right)+B\left(q^{-1}\right) R\left(q^{-1}\right)=K(q)
$$

This relation represents the Bezout equation already defined in the traditional approach of RST controller [13]. That proves that closedloop poles must be well optimized in order to satisfy the desired performances.

\subsection{Robustness}

In order to reject a static perturbation, the polynomial $S\left(q^{-1}\right)$ must have a term in $\left(1-q^{-1}\right)$. The introduction of a term of $\left(1+q^{-1}\right)$ in the polynomial $R\left(q^{-1}\right)$ makes possible the attenuation of the effect of noises in high frequencies on the entry of the system [1]-[2] and [15]. By taking in account these pre-specified fixed parts, polynomials $\tilde{R}\left(q^{-1}\right)$ and $\tilde{S}\left(q^{-1}\right)$ are then given by:

$$
\begin{aligned}
& \tilde{R}\left(q^{-1}\right)=H_{R}\left(q^{-1}\right) R\left(q^{-1}\right) \\
& \tilde{S}\left(q^{-1}\right)=H_{S}\left(q^{-1}\right) S\left(q^{-1}\right)
\end{aligned}
$$

where $H_{S}\left(q^{-1}\right)=1-q^{-1}$ and $H_{R}\left(q^{-1}\right)=1+q^{-1}$

The controller is then designed from the model governed by the following transfer function:

$$
\tilde{H}\left(q^{-1}\right)=\frac{B\left(q^{-1}\right) H_{R}\left(q^{-1}\right)}{A\left(q^{-1}\right) H_{S}\left(q^{-1}\right)}
$$

In order to maintain nominal performances in presence of disturbances and of modelling errors, the sensitivity functions defined below are evaluated and recomputed to satisfy the performances required. The sensitivity functions are given by [15]:

- Output disturbance: represented by the transfer function $S_{y d}\left(q^{-1}\right)$ between the output disturbance $d(t)$ and the output system $y(t)$ :
$S_{y d}\left(q^{-1}\right)=\frac{A\left(q^{-1}\right) \tilde{S}\left(q^{-1}\right)}{K\left(q^{-1}\right)}$

- Input disturbance: represented by the transfer function $S_{u d}\left(q^{-1}\right)$ between the output disturbance $d(t)$ and the input system $u(t)$ :

$S_{u d}\left(q^{-1}\right)=-\frac{A\left(q^{-1}\right) \tilde{R}\left(q^{-1}\right)}{K\left(q^{-1}\right)}$

The polynomial $K\left(q^{-1}\right)$ is given by the equation (30).

\subsection{Saturation effects}

The additive static disturbances cause generally the increase in the control signal magnitude applied to the system. Consequently, it is necessary to design a device of anti-saturation according to the technique already developed in [15] and exploited in [13] where the control law has the following forms:

$u_{k}=K(q) z_{k}^{d}-\tilde{R}\left(q^{-1}\right) y_{k}-\bar{S}\left(q^{-1}\right) u_{k-1}$

with:

$\tilde{S}\left(q^{-1}\right)=1+q^{-1} \bar{S}\left(q^{-1}\right)$

In addition, it is possible to impose certain dynamics when the system leaves the saturation. The desired dynamics is defined by the polynomial $P_{S}\left(q^{-1}\right)$ given by equation (38).

$P_{S}\left(q^{-1}\right)=1-\exp \left(-\frac{T_{e}}{\tau_{s a t}}\right) q^{-1}$

$\tau_{\text {sat }}$ indicates a time-constant of a system chosen of a first order.

Finally, the new control law of the flatnessbased polynomial controller in the presence of saturation is given by the expression:

$$
\begin{aligned}
P_{S}\left(q^{-1}\right) u_{k} & =K(q) z_{k}^{d}-\tilde{R}\left(q^{-1}\right) y_{k}- \\
& -\left(\tilde{S}\left(q^{-1}\right)+\exp \left(-\frac{T_{e}}{\tau_{\text {sat }}}\right)\right) \bar{u}_{k-1}
\end{aligned}
$$

with :

$$
\bar{u}_{k}= \begin{cases}u_{k} & \text { if } u_{\min } \leq u_{k} \leq u_{\max } \\ u_{\max } & \text { if } u_{k}>u_{\max } \\ u_{\min } & \text { if } u_{k}<u_{\min }\end{cases}
$$


$u_{\min }$ and $u_{\max }$ are respectively the lower and higher limits of the control saturation.

\section{Structure of Multiple Flatness- based Controllers}

The realisable structure of the flatness-based RST multi-controllers can be obtained by:

$S_{j}\left(q^{-1}\right) u_{k, j}=K(q) z_{k, j}^{d}+R_{j}\left(q^{-1}\right) y_{k, j}$

where:

$$
\begin{aligned}
R_{j}\left(q^{-1}\right) & =-(a-k) \mathbf{A}_{j}^{n-1} O^{-1}{ }_{\left(\mathbf{A}_{j}, \mathbf{C}_{j}\right)} Q \\
S\left(q^{-1}\right) & =1+(a-k) \cdot \\
& \cdot\left(\mathbf{A}_{j}^{n-1} O_{\left(\mathbf{A}_{j}, \mathbf{C}_{j}\right)}^{-1} M_{\left(\mathbf{A}_{j}, \mathbf{B}_{j}, \mathbf{C}_{j}\right)}-\right. \\
& \left.-\left(\mathbf{A}_{j}^{n-2} \mathbf{B}_{j} \ldots \mathbf{B}_{j}\right)\right) Q^{*}
\end{aligned}
$$

where $z_{k, j}^{d}$ are the flat output of each model $G_{j}$.

The design of flatness-based multi-controllers is presented in Figure 4.

\section{Application to the Control of a Thermal Process}

\subsection{Thermal process models}

The considered system is a thermal process defined in [7]. The flow rate signal is assumed piecewise constant and can vary by changing the throttle position; the transfer function of the model is given by:

$G_{j}(p)=\frac{k_{j} e^{-\tau_{j} p}}{1+T_{c j} p}$

where $k_{j}$ is $j^{\text {th }}$ static gain and $\tau_{j}$ is the $j^{\text {th }}$ delay, $T_{c j}$ is the $j^{\text {th }}$ time constant of the process and $p$ is the Laplace operator. Three operating points have been chosen to carry out the study.

All continuous-time models are sampled at $T_{e}=0.3 \mathrm{~s}$, leading to the structure for the corresponding discrete-time transfer functions given bv 「71:

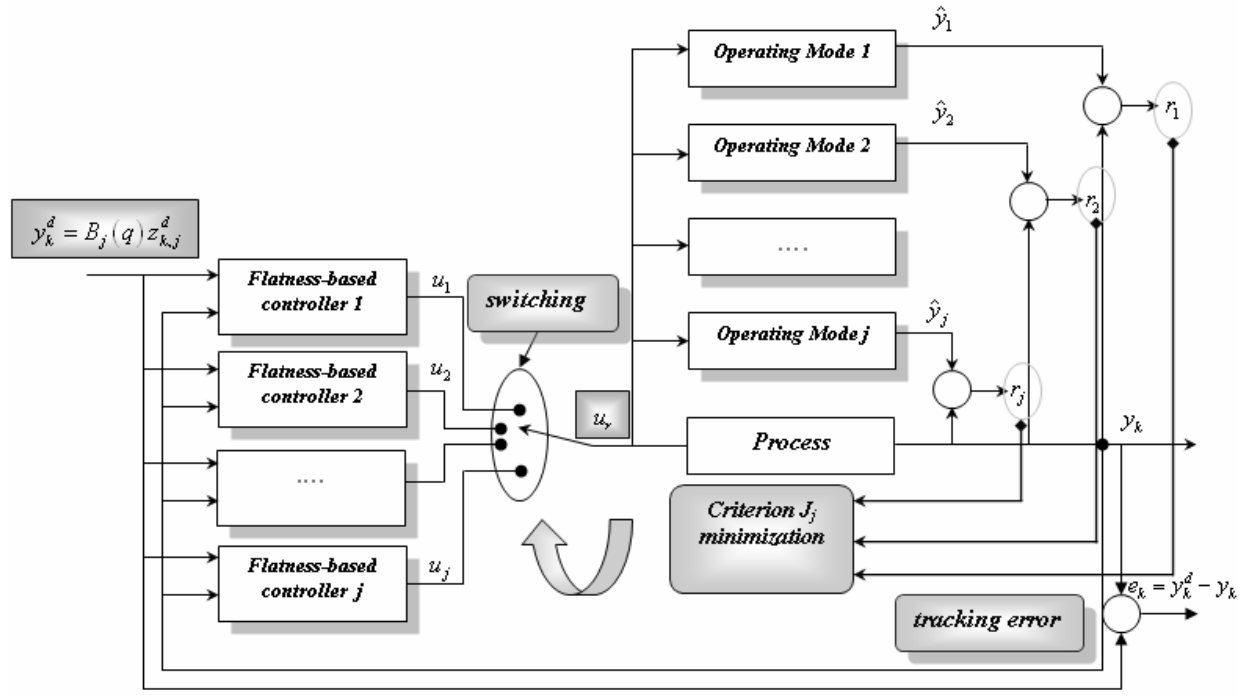

Figure 4. Robust Flatness-based multi-controllers reconfiguration

The dynamics of the closed-loop is defined by the tracking polynomial $K\left(q^{-1}\right)$ such as:

$$
\begin{aligned}
& A_{j}\left(q^{-1}\right) S_{j}\left(q^{-1}\right)+B_{j}\left(q^{-1}\right) R_{j}\left(q^{-1}\right)=K\left(q^{-1}\right) \\
& \left\{\begin{array}{c}
S_{1}\left(q^{-1}\right) u_{k, 1}=K(q) z_{k, 1}^{d}+R_{1}\left(q^{-1}\right) y_{k, 1} \\
S_{2}\left(q^{-1}\right) u_{k, 2}=K(q) z_{k, 2}^{d}+R_{2}\left(q^{-1}\right) y_{k, 2} \\
\vdots \\
S_{j}\left(q^{-1}\right) u_{k, j}=K(q) z_{k, j}^{d}+R_{j}\left(q^{-1}\right) y_{k, j}
\end{array}\right.
\end{aligned}
$$

$$
G_{1}\left(q^{-1}\right)=\frac{0.0510 q^{-1}+0.3427 q^{-2}}{1-0.5421 q^{-1}}
$$$$
G_{2}\left(q^{-1}\right)=\frac{0.121 q^{-1}+0.207 q^{-2}}{1-0.528 q^{-1}}
$$ 


\subsection{Trajectories planning}

In the discrete-time framework, the desired output trajectory $y_{k}^{d}$ is obtained from the equation (21).

In our case of study, the trajectories $z_{j}^{d}(t)$, $j=1,2,3$, are expressed in a continuous-time domain according to the following polynomial form:

$$
z_{j}^{d}(t)= \begin{cases}\frac{y_{j}^{d}\left(t_{0}\right)}{B_{j}(1)}, & \text { if } 0 \leq t \leq t_{0} \\ \text { Poly }(t), & \text { if } t_{0} \leq t \leq t_{f} \\ \frac{y_{j}^{d}\left(t_{f}\right)}{B_{j}(1)}, & \text { if } t \geq t_{f}\end{cases}
$$

where $B_{j}(1)$ is calculated for each operating mode.

The transition times are chosen $t_{0}=10 \mathrm{~s}$ and $t_{f}=20 \mathrm{~s}$ and the polynomial Poly $(t)$ a reference trajectory between these two moments:

$$
\operatorname{Poly}(t)=\left(\begin{array}{lll}
1 & 0 & 0
\end{array}\right)\left(M_{1}\left(t-t_{0}\right) c_{1}+M_{2}\left(t-t_{0}\right) c_{2}\right)
$$

where $c_{1}$ and $c_{2}$ vectors are given by:

$$
\begin{aligned}
& c_{1}=Z^{d}\left(t_{0}\right) \\
& c_{2}=M_{2}^{-1}\left(t_{f}-t_{0}\right)\left(Z^{d}\left(t_{f}\right)-M_{1}\left(t_{f}-t_{0}\right) Z^{d}\left(t_{0}\right)\right)
\end{aligned}
$$

with $M_{1}(t)$ and $M_{2}(t)$ defined in [13].

The roots of the desired polynomial $K(q)$ are: $p_{1,2}=0.5087 \pm 0.3019 i$ and $p_{3}=0.2231$.

The desired trajectories $z_{1}^{d}(t), z_{2}^{d}(t)$ and $z_{3}^{d}(t)$ are then represented on Figure 5.

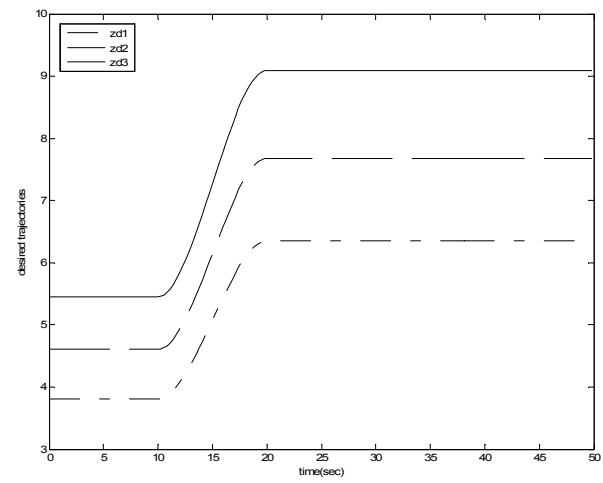

Figure 5. The desired flat output trajectories

$$
z_{1}^{d}(t), z_{2}^{d}(t) \text { and } z_{3}^{d}(t)
$$

\subsection{Stability using LMI}

The LMI problem implies to find a common Lyapunov function for the switched linear system. Hence, the optimization problem is feasible and the overall system, i.e., the combination of the three controllers with the three process transfer functions, is found to be globally asymptotically stable. The definite positive matrix is given by:

$$
\mathbf{P}=\left(\begin{array}{ll}
59.5503 & -19.6844 \\
-19.6844 & 19.5066
\end{array}\right)
$$

The $\mathbf{L}_{j}$ observer's gains calculated in closedloop using LMI are:

$$
\begin{aligned}
& \mathbf{L}_{1}=\left(\begin{array}{l}
1.3336 \\
2.5711
\end{array}\right), \mathbf{L}_{2}=\left(\begin{array}{l}
0.3140 \\
0.7804
\end{array}\right) \text { and } \\
& \mathbf{L}_{3}=\left(\begin{array}{l}
-0.5536 \\
-1.0366
\end{array}\right)
\end{aligned}
$$

\subsection{Simulation results}

The flatness-based RST switching multimulti-controllers used for the thermal process is described in Figure 6.

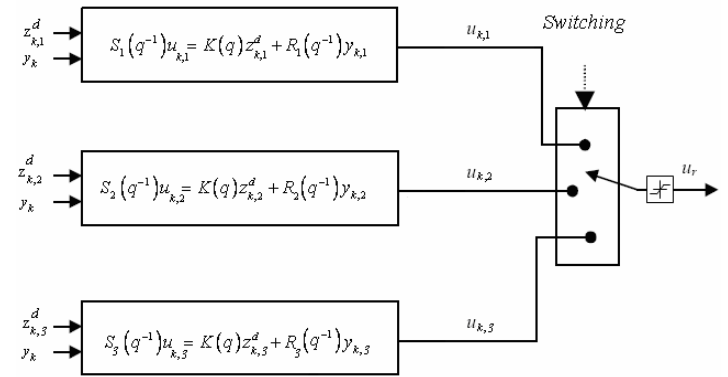

Figure 6. Flatness-based RST switching multi-controllers

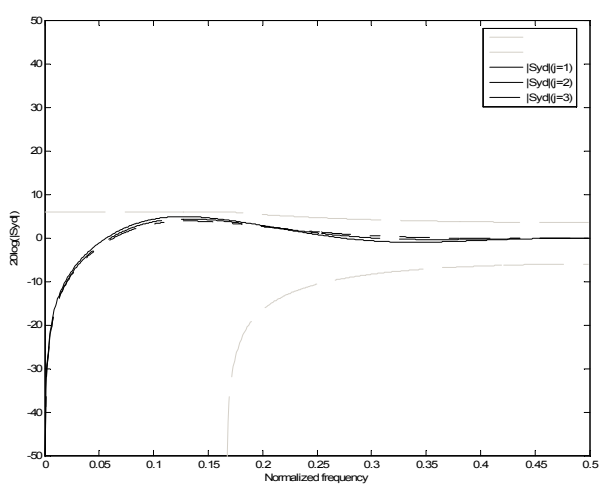

(a) Output sensitivity function $\left|S_{y d}\right|$ 


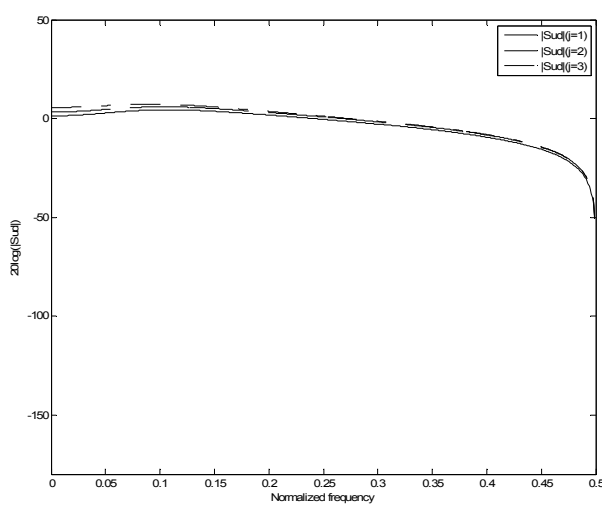

(b) Input sensitivity function $\left|S_{u d}\right|$

Figure 7. Sensitivity functions for $j=1,2,3$

The results of Figure 7 show that the multicontrollers obtained are robust considering the sensitivity functions which remain inside the specified templates of robustness. In addition, we underline the robustness of such multi-controllers related to the static disturbance and the high frequency noises rejection is clearly guaranteed.

The simulation results for the case of thermal process are shown in Figure 8.

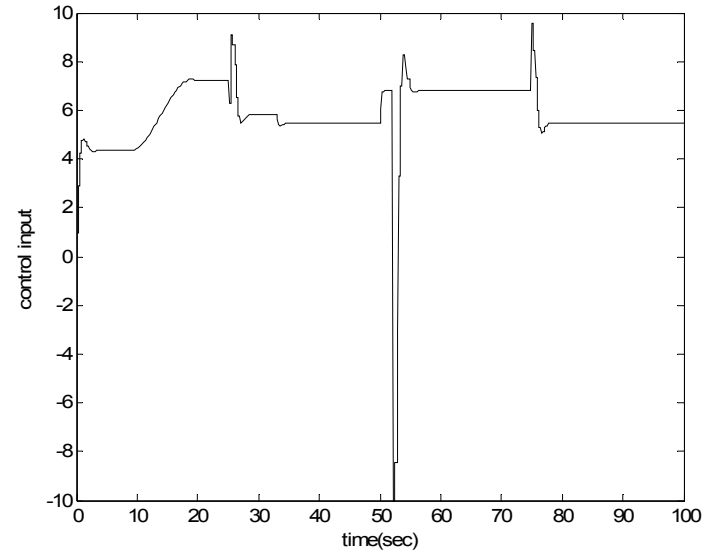

(a) Control input

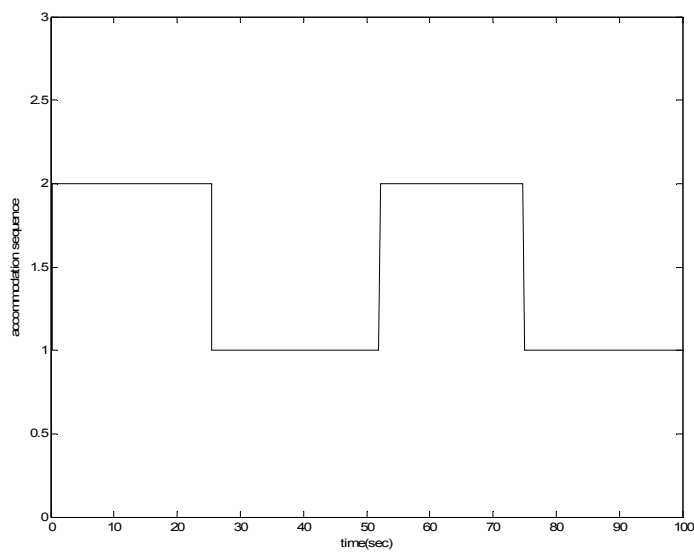

(b) Switching sequence of accommodation signal

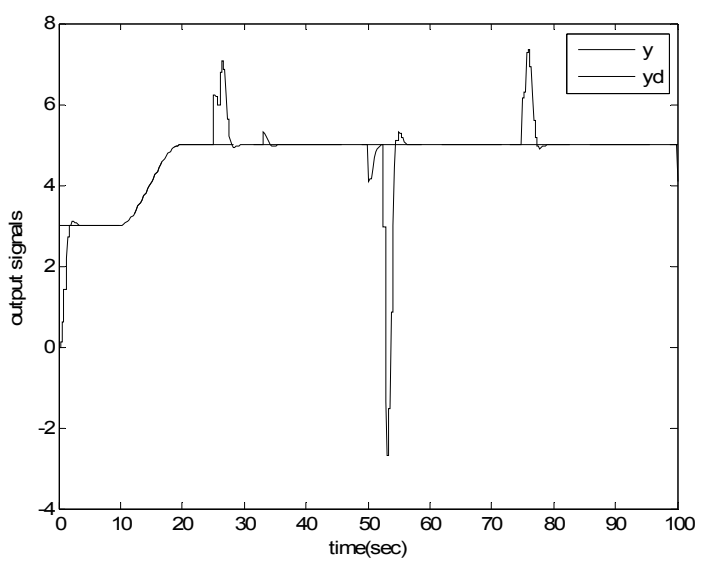

(c) System output y and desired output

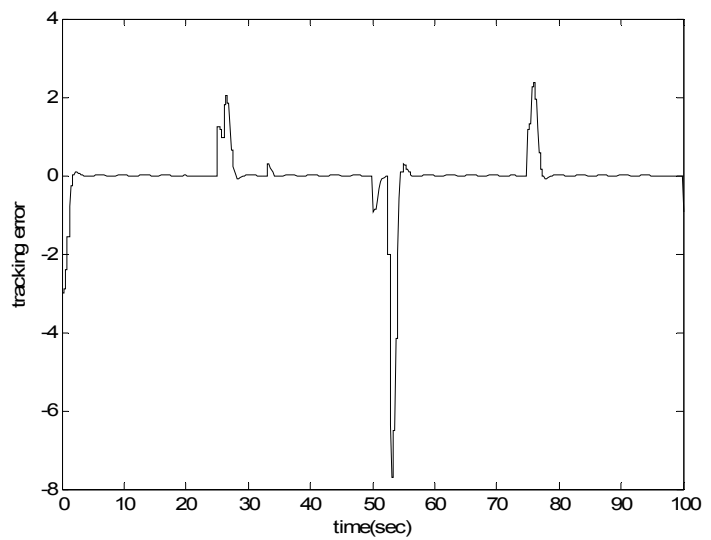

(d) Tracking error

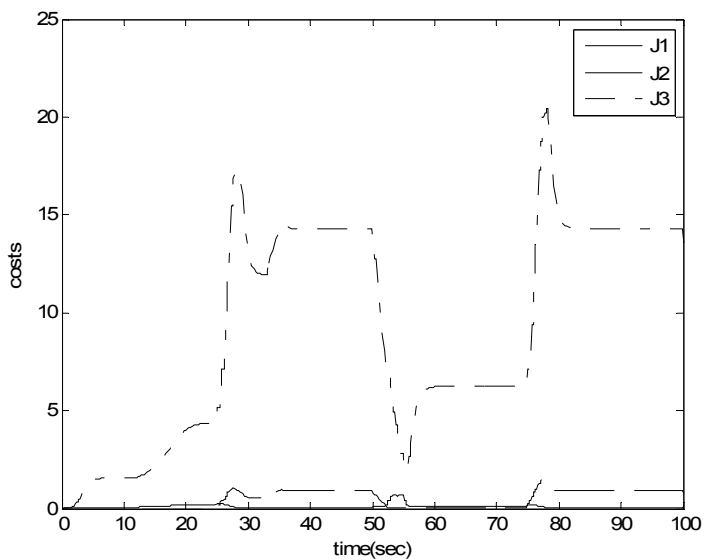

(e) Criterion signals

Figure 8. Simulation results of robust flatness-based RST multi-controllers with anti-windup device

\section{Conclusion}

The proposed approach based on the flatness concept is applied to a thermal process with multiple operating modes. For each model, a corresponding RST flatness-based polynomial controller is designed and consequently, a reconfiguration strategy 
based on multi-controllers structure obtained. The robust reconfiguration flatness-based control is based on switching between identified operating mode of a thermal process based on the minimization of criterion value which is generated from a comparison between the estimated operating modes output and the real system output. The real thermal process is then controlled by a control signal which is issued from the active flatness-based RST multi-controllers. Satisfactory simulation results are obtained.

\section{REFERENCES}

1. AYADI, M., Contribution à la commande des systèmes linéaires plats de dimension finie, Thèse de Doctorat, Institut National Polytechnique de Toulouse, 2002.

2. AYADI, M., J. HAGGÈGE, S. BOUALLÈGUE, M. BENREJEB, A Digital Flatness Control System of a DC Motor, Studies in Informatics and Control, Vol. 17, 2008, pp. 205-224.

3. BENREJEB, M., M. GASMI, P. BORNE, New Stability Conditions for TS Fuzzy Continuous Nonlinear Models. Nonlinear Dynamics and Systems Theory. Vol. 5, 2005, pp. 369-376.

4. BLANKE, M., M. KINNAERT, J. LUNZE, M. STAROSWIECKI, Diagnosis and Fault-Tolerant Control, Springer, London, 2006.

5. BORNE, P., Multimodel Analysis and Control of Complex Systems, 1st SinoFrench Symposium on Sensory Evaluation and Intelligent Technique, Shangai, 2003.

6. BORNE, P., G. DAUPHIN-TANGUY, J. P. RICHARD, F. ROTELLA, I. ZAMBETTAKIS, Commande et optimisation des processus, Edition Technip, Paris, 1990.

7. CHARBONNAUD, P., J. F. CARRILO, S. MÉDAR, Robust Control Reconfiguration of a Thermal Process with Multiple Operating Modes, IEEE Transactions on Control Systems Technology, Vol. 11, 2003 pp. 529-538.
8. DELMOTTE, F. P. BORNE, A General Scheme for Multi-model Controller Using Trust, Mathematics and Computers in Simulation, Vol. 41, 1996, pp. 173-186.

9. DING, X. S., Model-based Fault Diagnosis Techniques Design Schemes, Algorithm, and Tools, Springer, London, 2003.

10. DUVIELLA, E., P. CHIRON, P. CHARBONNAUD, Networked Hydrographical Systems: A Reactive Control Strategy Integrating Time Transfer Delays, Studies in Informatics and Control, Vol. 17, 2008, pp.153-168.

11. ELGHAOUI, L., S. NICULESCU, Advances in Linear Matrix Inequality methods in control, Philadelphia, 2000.

12. FLIESS, M., J. LÉVINE, PH. MARTIN P. ROUCHON, On differentially flat non linear systems, IFAC Symposium NOLLOS'92, 1992, pp. 408-412.

13. GHARSALLAOUI, H., M. AYADI, M. BENREJEB, P. BORNE, Flatness-based Control and Conventional RST Polynomial Control of a Thermal Process, International Journal of Computers, Communications \& Control, Vol. 4, 2009, pp. 27-41.

14. GHARSALLAOUI, H., M. AYADI, M. BENREJEB, P. BORNE, Flatness-based Switching Control, 12th LSS 2010 IFAC Symposium on Large Scale Systems: Theory and Applications, Lille, 2010.

15. LANDAU, I. D., R. LOZANO, M. M'SAAD, Adaptive Control, SpringerVerlag, London, 1998.

16. LEMOS, J., L. RATOS, J. MARQUES, Switching Reconfigurable Control Based on Hidden Markov models, American Control Conference, San Diego, 1999.

17. MAHMOUD, M., J. JIANG, Y. ZHANG, Stochastic Stability Analysis of Fault Tolerant Control Systems in the Presence of Noise, IEEE Transactions on Automatic Control, Vol. 11, 2001, pp. 1810-1815.

18. MÉDAR, S., P. CHARBONNAUD, F. NOUREDINE, Active Fault 
Accommodation of a Three Tank System via Switching Control, IFAC World Congress, Barcelona, 2002.

19. MENDOZA, J. E., L. A. VILLALEIVA, M. A. CASTRO, E. A. LOPEZ, Multiobjective Evolutionary Algorithms for Decision-Making in Reconfiguration Problems Applied to the Electric Distribution Networks, Studies in Informatics and Control, Vol. 18, 2009, pp. 325-336.

20. NARENDRA, K. S., O. A. DRIOLLET, M. FEILER, K. GEORGE, Adaptive Control using Multiple Models, Switching and Tuning, International Journal of Adaptive Control and Signal Processing, Vol. 17, 2003, pp. 87-102.

21. PATTON, R. J., Fault-tolerant Control: the 1997 Situation, Preprints of IFAC Symposium on Fault Detection Supervision and Safety for Technical Processes, Kingston upon Hull, UK, 1997.

22. RODRIGUES, M., Diagnostic et commande active tolérante aux défauts appliqués aux systèmes décrits par des multi-modèles linéaires, Thèse de Doctorat, Université Henri Poincaré, Nancy 1, 2005.
23. ROTELlA, F., F. CARILlO, M. AYADI, Digital Flatness Based Robust Controller Applied to A Thermal Process, IEEE International Conference on Control Applications, Mexico, 2001, pp. 936-941.

24. STEFFEN, T., Control Reconfiguration of Dynamical Systems, Springer, 2005.

25. STAROSWIECKI, M., Fault Tolerant Control: The Pseudo-Inverse Method Revisited, Proceedings of the 16th IFAC World Congress, Prague, 2005.

26. ZHANG, Y., J. JIANG, Bibliographical Review on Reconfigurable Faulttolerant Control Systems, Proceeding of the SAFEPROCESS: 5th IFAC Symposium on Detection and Safety for Technical Processes, Washington, 2003. 
